\title{
Estudio del comportamiento a cortante de vigas de hormigón reforzado con fibras
}

\section{Study of the shear behaviour of fibre reinforced concrete beams}

\author{
$\underline{\text { J. Turmo }}^{(*)}$, N. Banthia ${ }^{(* *)}$, R. Gettu(***) y B. Barragán ${ }^{(* * * *)}$
}

Recepción/Received: 5-III-07

Aceptación/Accepted: 3 -VII-07

Publicado online/Online publishing: 30-V-08

\section{RESUMEN}

Este trabajo presenta una serie de ensayos para caracterizar el comportamiento estructural de vigas realizadas con hormigón reforzado con fibras sometidas a cortante. El programa de ensayos incluía tres tipos de fibras, dos de acero y una de polipropileno. Asimismo, se realizó una serie de ensayos con una viga confeccionada con hormigón armado convencional. La resistencia a cortante de las vigas es comparada con los valores que la formulación existente predice. El estudio confirma que la tenacidad y la resistencia a cortante son incrementadas tras la adición de fibras al hormigón. Sin embargo, la incorporación de un $1 \%$ en volumen de fibras conduce a valores de resistencia última a cortante inferiores a los obtenidos con vigas de hormigón convencional con la misma cantidad de acero dispuesta en forma de cercos de cortante. Los actuales métodos de cálculo parecen lo suficientemente precisos para evaluar la carga de cortante último, incluso cuando los parámetros mecánicos utilizados en las fórmulas (tenacidad, resistencia a tracción) son deducidos a partir de la formulación propuesta en la normativa.

Palabras clave: hormigón, refuerzo de fibras, resistencia a la flexión, resistencia a la compresión, propiedades mecánicas.

\section{SUMMARY}

This study presents a series of tests for characterizing the structural behaviour of fibre reinforced concrete beams subjected to shear loading. The experimental program involves three types of fibres; two steel fibres and a polypropylene fibre. As a reference, plain concrete and conventionally reinforced concrete specimens have also been tested. The ultimate shear capacity of the beams is calculated and these values compared with those predicted by existing formulations. The study confirms that the toughness and shear crack resistance of the material is greatly enhanced by the fibres. However, the incorporation of $1 \%$ of fibres yielded lower shear strength than conventionally reinforced beams with the same amount of steel in the form of transversal stirrups. Existing design methods seem sufficiently robust to estimate the maximum shear load, even when using material properties (toughness, tensile strength) extrapolated from code formulae.

Keywords: concrete, fibre reinforcement, flexural strength, compressive strength, mechanical properties.

\footnotetext{
(*) Universidad de Castilla La Mancha (Ciudad Real, España).

(**) University of British Columbia (Vancouver, Canada).

(***) Indian Institute of Technology Madras (Chennai, India).

$(* * * *)$ BASF Construction Chemicals España, S.L. (Barcelona, España).
} 


\section{INTRODUCCIÓN}

Las fibras metálicas y poliméricas son usadas para reforzar el hormigón e incrementar su tenacidad y mejorar el control de la fisuración. El hormigón reforzado con fibras (Fibre Reinforced Concrete, FRC) puede ser usado reduciendo o incluso eliminando la armadura convencional. En nuestro país, el mayor campo de aplicación del FRC corresponde al hormigón proyectado y a pavimentos (1). Uno de los fines para los que el FRC es usado es para incrementar la resistencia de elementos sometidos a cortante. Son numerosas las formulaciones propuestas para cuantificar el incremento de resistencia a cortante que induce la introducción de fibras en el hormigón (2-7). Cada una de ellas es función de un índice que tiene en cuenta de alguna manera la tenacidad del material. Cada una de estas fórmulas emplea un índice diferente que se obtiene a partir de distintos tipos de ensayos, sobre diferentes tipos de probetas, todos ellos perfectamente definidos. Sin embargo, debido a que en general no se dispone de tanta información sobre el material, la aplicación de estas fórmulas en proyecto se ve dificultada. Además, muchas de estas fórmulas están diseñadas sólo para la evaluación de hormigones reforzados con fibras metálicas (Steel Fibre Reinforced Concrete, SFRC).

El propósito de esta comunicación es presentar una serie de ensayos realizados para caracterizar el comportamiento estructural a cortante de vigas de hormigón reforzado con fibras de diferentes formas y materiales (acero y polipropileno). Además, se evalúa hasta qué punto se puede llegar a predecir la respuesta al cortante último de una viga de FRC extrapolando los índices usados en las fórmulas a partir de la información que se tenga disponible del material. Asimismo, se comprueba si la formulación obtenida para el hormigón reforzado con fibras de acero es extrapolable a un hormigón reforzado con fibras de polipropileno.

\section{MECANISMOS DE RESISTENCIA A COR- TANTE DE LAS VIGAS DE FRC}

En una viga de FRC parece lógico pensar que, en rotura, la fuerza de cortante transferida por el alma debido al efecto engranaje de los áridos se incrementa, ya que ésta depende de la apertura de fisura (o de la apertura relativa de la fisura con respecto al tamaño de los áridos) y con el FRC el tamaño de la fisura se ve claramente reducido. Además, debido al diferente comportamiento postpico del FRC, una vez producida la fisura de cortante existen unas tensiones de tracción atravesando la fisura cuya proyección vertical equilibra el cortante que actúa sobre la viga. Estas tensiones de tracción equivalentes al esfuerzo necesario para arrancar las fibras de la matriz de

\section{INTRODUCTION}

Both steel and polymeric fibres have been used to reinforce concrete and consequently increase its toughness and crack resistance. Fibre reinforced concrete (FRC) can be used in some structural applications with a reduced amount or even without any conventional bar reinforcement, as in shotcreting or paving construction (1). A promising application of the fibres is to increase the loadcarrying capacity of concrete subjected to shear. Several design methods have been proposed that take into account the increase in shear strength due to the fibres (2-7). Each of the methods accounts for the fibre contribution by means of an index based on the toughness of the material. However, each formula uses a different index, obtained from different types of test configurations. Since usually not that much information of the material is available, the application of the design methods can be difficult. Moreover, most of the design methods and tests procedures have been developed only for the evaluation of steel fibre reinforced concrete (SFRC).

The purpose of this work is to present the results of a study carried out to characterize the structural behaviour of FRC beams under shear loading, considering fibres of different shapes and materials (steel and polymeric). Further, the study aims to evaluate up to what extent the ultimate shear capacity can be predicted by the existing design method when toughness parameters are extrapolated from the available material information. At the same time, it is verified whether the design methods for SFRC can be extrapolated to polypropylene fibre reinforced concrete.

\section{SHEAR RESISTANT MECHANISMS OF FRC BEAMS}

In an FRC beam, it seems logical to consider that, during failure, the shear force transferred along the web, due to the aggregate interlocking, is increased since the crack opening is reduced by the fibre action. Also, due to the toughness of FRC, tensile stresses can be resisted across the crack once the shear crack appears (through fibre bridging), giving rise to a vertical force component that contributes to balancing the shear force acting on the beam. These tensile stresses, necessary to pullout the 
hormigón son las que definen el comportamiento postfisuración del material.

El método que propugnan las normas europeas y el código ACI americano para la evaluación de la respuesta a cortante último Vu consiste en sumar un término que recoge la contribución del hormigón Vcd y otro que representa el papel de los cercos Vwd en la resistencia a cortante.

$$
\mathrm{V}_{\mathrm{u}}=\mathrm{V}_{\mathrm{cd}}+\mathrm{V}_{\mathrm{wd}}
$$

Siguiendo esta línea, muchos autores (2-5) sugieren para FRC la adición de otro sumando Vfd, que recoge el efecto de las fibras:

$$
\mathrm{V}_{\mathrm{u}}=\mathrm{V}_{\mathrm{cd}}+\mathrm{V}_{\mathrm{wd}}+\mathrm{V}_{\mathrm{fd}}
$$

Accordingly, several authors (2-5) have suggested the addition of another factor, Vfd, to take into account the fibre contribution:

donde $V_{\mathrm{fd}}$ es función de diversos parámetros. Así, a modo de ejemplo, según RILEM (5), la contribución de la fibra metálica viene dada por:

$$
V_{f d}=k_{f} k_{1} \tau_{f d} b_{w} d
$$

where $V_{f d}$ is a function of different parameters. For example, RILEM (5) defines the contribution of steel fibres to the ultimate shear capacity as:

$\tau_{\mathrm{fd}}=$ valor de diseño de la tensión tangencial máxima debida a las fibras: $\tau_{\mathrm{fd}}=0.12 \cdot \mathrm{f}_{\text {eqk }, 3}(\mathrm{MPa})$ donde $\mathrm{f}_{\text {eqk }, 3}$ es un valor de resistencia equivalente obtenido del ensayo de flexotracción normalizado por RILEM (8).

$\tau_{f d}=$ design value of the maximum tangential stress due to fibres; $\tau_{f d}=0.12 \cdot f_{e q k, 3}(\mathrm{MPa})$; where feqk,3 is the equivalent residual stress from the bending test recommended by RILEM (8).

donde: $\mathrm{k}_{\mathrm{f}}=$ factor para tener en cuenta la contribución de las alas en una sección en $\mathrm{T}$ :

where $k_{f}$ takes into account the contribution of the flanges of a T-section:

$$
\begin{aligned}
\mathrm{k}_{\mathrm{f}}=1+\left(\frac{h_{f}}{b_{w}}\right)\left(\frac{h_{f}}{d}\right) \mathrm{n}, \mathrm{k}_{\mathrm{f}} & \leq 1.5 \\
h_{f} & =\text { flange thickness }(\mathrm{mm}) \\
b_{f} & =\text { flange width }(\mathrm{mm}) \\
b_{w} & =\text { web width }(\mathrm{mm})
\end{aligned}
$$

$\mathrm{h}_{\mathrm{f}}=$ espesor del ala $(\mathrm{mm})$

$b_{f}=$ anchura del ala $(\mathrm{mm})$

$\mathrm{b}_{\mathrm{w}}=$ anchura del alma $(\mathrm{mm})$

$$
\mathrm{n}=\frac{b_{f}-b_{w}}{h_{f}} ; \mathrm{n} \leq 3 ; \mathrm{n} \leq \frac{3 b_{w}}{h_{f}}
$$

$\mathrm{Y} \mathrm{k}_{1}$ tiene en cuenta el efecto escala

And $k_{1}$ accounts for the size effect

$$
\mathrm{k}_{1}=\frac{1600-d}{1000} \quad \mathrm{k}_{1} \geq 1 ;(d \text { in } \mathrm{mm}) ;
$$

\section{MATERIALES}

La dosificación empleada como referencia para el hormigón sin fibras (Tabla 1) tenía como meta alcanzar una resistencia de $50 \mathrm{MPa}$. Para los FRC se usaron tres tipos de fibras, todas ellas de longitud $50 \mathrm{~mm}$, fibras de acero con extremos deformados Dramix RL 45/50 BN, fibras de acero Novotex 1050 con extremos achatados y fibras de polipropileno onduladas S-152 HPP. La cantidad de las mismas en la mezcla era de un $1 \%$ en volumen, lo cual puede considerarse como un límite práctico superior de contenido de fibras. Un resumen de las piezas hormigonadas aparece en la Tabla 2.

\section{MATERIALS}

The base mix composition, Table 1, aimed to achieve a compressive strength of $50 \mathrm{MPa}$. Three types of fibres were considered, all with $50 \mathrm{~mm}$ length: Dramix 45/50 $B N$ hook-ended steel fibres, Novotex 1050 flat-ended steel fibres, and S-152 HPP ondulated polypropylene fibres. In all cases the fibre volume was fixed at $1 \%$, which can be considered as the upper limit due to practical reasons, such as ease of mixing and placing. Table 2 summarizes the cast specimens. 
Tabla 1 / Table 1

Dosificación del hormigón.

Mix proportions.

\begin{tabular}{|c|c|}
\hline Componente/Component & $\mathbf{K g} / \mathbf{~ m}^{3}$ \\
\hline Cemento/Cement & 363 \\
\hline Arena/Sand & 804 \\
\hline Grava/Gravel 10 mm MSA & 579 \\
\hline Grava/Gravel 20 mm MSA & 464 \\
\hline Agua/Water & 181 \\
\hline Superplastificante/Superplastizicer (Its/m³) & 2,0 \\
\hline
\end{tabular}

Tabla 2 / Table 2

Elementos hormigonados.

Specimen details.

\begin{tabular}{|c|c|c|c|}
\hline Hormigón/Concrete & & & Probetas / Cast specimens \\
\hline $\begin{array}{l}\text { PC-NSR (referencia/ } \\
\text { reference) }\end{array}$ & \begin{tabular}{|l|}
3 cilindros/cylinders \\
$100 \times 200 \mathrm{~mm}$ \\
\end{tabular} & \begin{tabular}{|l|}
3 vigas/beams \\
$100 \times 100 \times 350 \mathrm{~mm}$
\end{tabular} & $\begin{array}{l}2 \text { vigas / beams } 96 \times 125 \times 1000 \mathrm{~mm} \text { Armado/Reinforcement: } \\
\text { longitudinal/ longitudinal } 2-\Phi 16(15 \mathrm{M}) \text {, transversal: ninguno/stirrups: none }\end{array}$ \\
\hline Dramix 1\% & $\begin{array}{l}3 \text { cilindros/cylinders } \\
100 \times 200 \mathrm{~mm}\end{array}$ & $\begin{array}{l}3 \text { vigas } / \text { beams } \\
100 \times 100 \times 350 \mathrm{~mm}\end{array}$ & $\begin{array}{l}2 \text { vigas / beams } 96 \times 125 \times 1000 \mathrm{~mm} \text { Armado/Reinforcement: } \\
\text { longitudinal/ longitudinal } 2-\Phi 16(15 \mathrm{M}) \text {, transversal: ninguno/stirrups: none }\end{array}$ \\
\hline Novotex 1050 1\% & $\begin{array}{l}3 \text { cilindros/cylinders } \\
100 \times 200 \mathrm{~mm}\end{array}$ & $\begin{array}{l}3 \text { vigas / beams } \\
100 \times 100 \times 350 \mathrm{~mm}\end{array}$ & $\begin{array}{l}2 \text { vigas / beams } 96 \times 125 \times 1000 \mathrm{~mm} \text { Armado/Reinforcement: } \\
\text { longitudinal/ longitudinal } 2-\Phi 16(15 \mathrm{M}) \text {, transversal: ninguno/stirrups: none }\end{array}$ \\
\hline HPP $1 \%$ & \begin{tabular}{|l|}
3 cilindros/cylinders \\
$100 \times 200 \mathrm{~mm}$ \\
\end{tabular} & $\begin{array}{l}3 \text { vigas / beams } \\
100 \times 100 \times 350 \mathrm{~mm}\end{array}$ & $\begin{array}{l}2 \text { vigas / beams } 96 \times 125 \times 1000 \mathrm{~mm} \text { Armado/Reinforcement: } \\
\text { longitudinal/ longitudinal } 2-\Phi 16(15 \mathrm{M}) \text {, transversal: ninguno/stirrups: none }\end{array}$ \\
\hline Control PC-SR & $\begin{array}{l}3 \text { cilindros/cylinders } \\
100 \times 200 \mathrm{~mm}\end{array}$ & $\begin{array}{l}3 \text { vigas/beams } \\
100 \times 100 \times 350 \mathrm{~mm}\end{array}$ & $\begin{array}{l}2 \text { vigas / beams } 96 \times 125 \times 1000 \mathrm{~mm} \text { Armado/Reinforcement: } \\
\text { longitudinal/ longitudinal } 2-\Phi 16(15 \mathrm{M}) \text {, transversal/stirrups: c } 6 / 50 \mathrm{~mm}\end{array}$ \\
\hline
\end{tabular}

\section{ENSAYOS DE CARACTERIZACIÓN DEL MATERIAL}

Para la caracterización del material usado en los ensayos a cortante se ensayaron probetas cilíndricas de $100 \times 200$ $\mathrm{mm}$ a compresión y probetas prismáticas $100 \times 100 \times 350$ $\mathrm{mm}$ a flexotracción con carga en tercios de luz. Los ensayos de caracterización del material se realizaron siguiendo las recomendaciones de la Japan Society of Civil Engineers (JSCE) para SFRC y la normativa ASTM. LoS ensayos a compresión de hormigones con fibras, se efectuaron siguiendo el artículo JSCE-SF5 (9), que además de la resistencia a compresión evalúa la tenacidad a compresión. Los ensayos de flexotracción se realizaron siguiendo la norma ASTM C1018 (10). La configuración del ensayo y la media de los resultados pueden verse en las Figuras 1 y 2 ; y 3 y 4 , respectivamente.

Como se deduce de las Figuras 3 y 4, el material incrementa su tenacidad con la adición de fibras, en mayor grado con las fibras de acero con extremos conformados, seguidas de aquéllas con los extremos achatados y de las poliméricas. Sin embargo, la resistencia a compresión de los FRC es inferior a la del hormigón de referencia. Este efecto también ha sido observado por otros investigadores (11).

\section{MATERIAL-SCALE CHARACTERIZATION TESTS}

The concrete compressive strength was evaluated for each mix by tests on $100 \times 200 \mathrm{~mm}$ cylinders following the JSCE (9) recommendations for SFRC, where the toughness under compressive loading is also evaluated. Fourpoint loading flexural tests were conducted on beam specimens of $100 \times 100 \times 350 \mathrm{~mm}$ according to the ASTM C1018 (10) standard. The test configuration and typical mean responses can be observed in Figs. 1-2 and Figs. 34 , respectively.

As it can be seen from Figs. 3 and 4, toughness clearly increases with the incorporation of fibres; to a greater extent with hook-ended fibers, followed by flat-ended and polypropylene fibres. However, that compressive strength is slightly reduced in the case of FRCs, with respect to the reference concrete. This effect has also been found by other researchers (11). 
La curva tensión-deformación en compresión permite el cálculo de los índices de tenacidad presentados en la Tabla 3. Los índices de tenacidad a flexión, calculados a partir de la curva carga-desplazamiento del ensayo de flexión, están recogidos en la Tabla 4. Estos índices, valores convencionales de la medida de la tenacidad, permiten ordenar los materiales por tenacidad creciente siempre y cuando se respeten las condiciones de ejecución del ensayo.

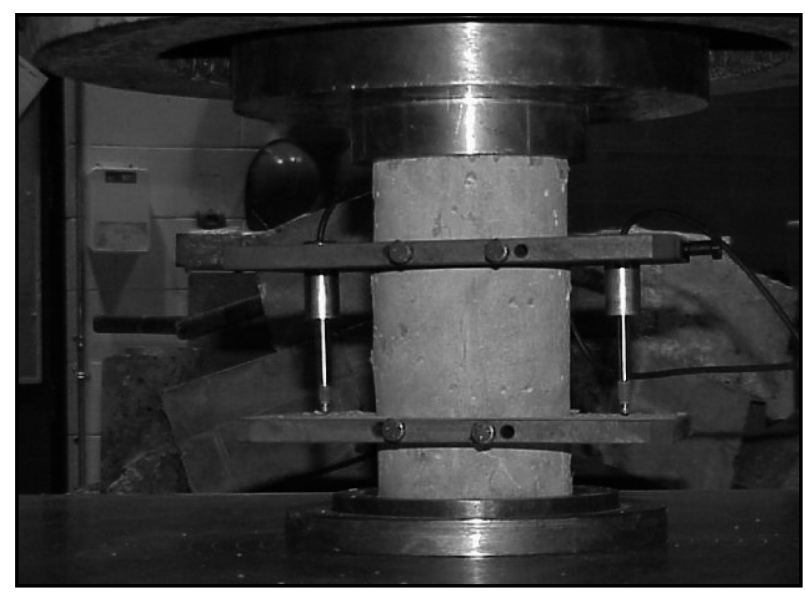

Figura 1. Configuración del ensayo de compresión.

Figure 1. Compression test configuration.

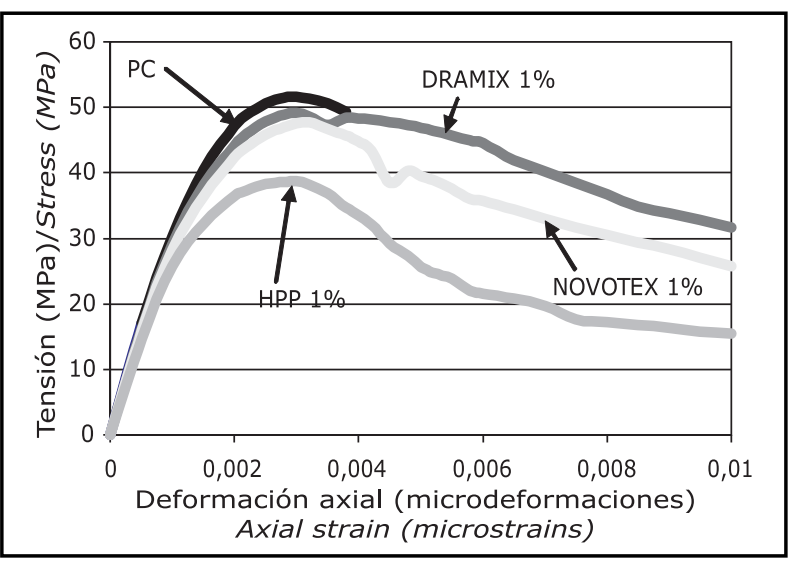

Figura 3. Diagramas tensión-deformación axil.

Figure 3. Stress-strain response.
The stress-strain response in compression permits the calculation of the toughness indices presented in Table 3. The flexural toughness indices calculated from the loaddisplacement response of a bending test, are presented in Table 4. These indexes permit the ranking of the materials in terms of toughness if standard test conditions are respected.

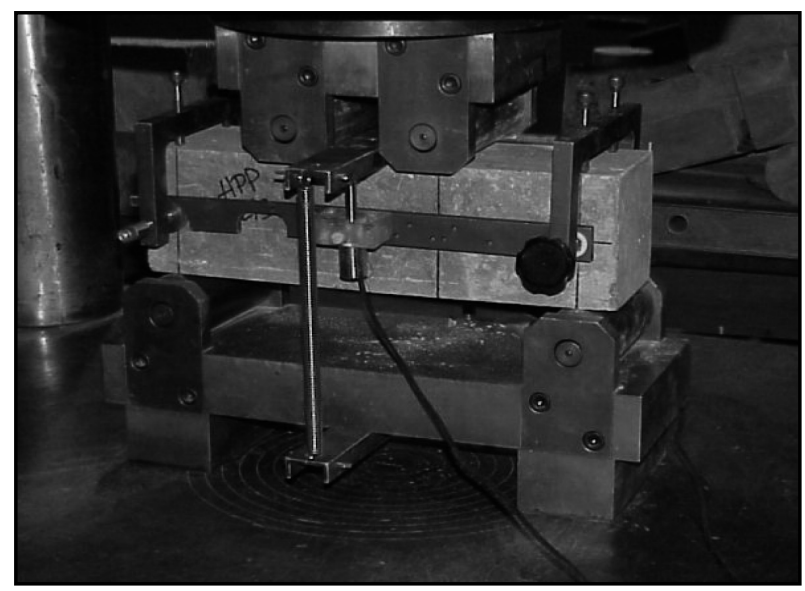

Figura 2. Configuración del ensayo de flexotracción. Figure 2. Bending test configuration.

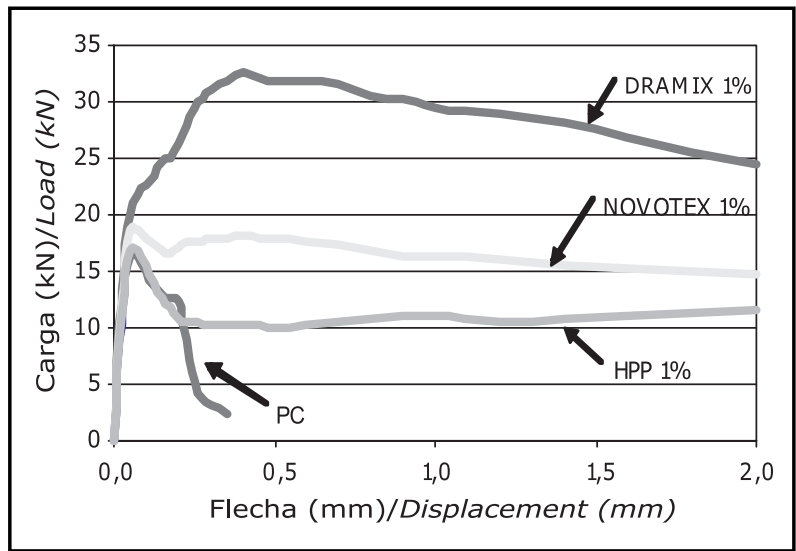

Figura 4. Diagramas carga-flecha ensayo tenacidad flexión. Figure 4. Load-displacement response.

Tabla 3 / Table 3

Resultados de ensayos a compresión. Resistencia y tenacidad JSCE. Strength and compressive toughness indices from JSCE standard.

\begin{tabular}{|l|c|c|c|c|}
\hline \multirow{2}{*}{ Parámetro/ Parameter } & PC & Dramix 1\% & Novotex 1\% & HPP 1\% \\
\cline { 2 - 5 } & $\mathrm{MPa}$ & $\mathrm{MPa}$ & $\mathrm{MPa}$ & $\mathrm{MPa}$ \\
\hline Resistencia/Strength (MPa) & 51.97 & 50.31 & 48.56 & 38.96 \\
\hline JSCE índice de tenacidad/JSCE toughness index & 21.08 & 40.51 & 38.11 & 32.57 \\
\hline
\end{tabular}


Tabla 4 / Table 4

Resultados del ensayo de flexotracción e índices de tenacidad a flexión ASTM C1018. Flexural toughness indices from ASTM C1018.

\begin{tabular}{|l|c|c|c|c|}
\hline $\begin{array}{l}\text { Parámetro/ Parameter } \\
\text { Índice/Index }\end{array}$ & P C & Dramix 1\% & Novotex 1\% & HPP 1\% \\
\hline Módulo de rotura (Mpa)/Modulus of rupture (MPa) & 4.97 & 9.83 & 6.09 & 5.18 \\
\hline I5 & $*$ & 4.67 & 5.01 & 4.62 \\
\hline I10 & $*$ & 10.29 & 9.78 & 9.07 \\
\hline I-20 & $*$ & 22.89 & 19.72 & 15.61 \\
\hline R5,10 & $*$ & 112.6 & 95.4 & 89 \\
\hline R10,20 & $*$ & 126.0 & 99.3 & 65.4 \\
\hline JSCE(MPa) & $*$ & 8.44 & 4.81 & 3.3 \\
\hline
\end{tabular}

\section{ENSAYOS ESTRUCTURALES Y ANÁLISIS DE RESULTADOS}

Las vigas fueron ensayadas a cortante bajo dos puntos de carga situados a tercios de luz, esto es, con una relación luz de cortante canto de la viga de 2,4 (Figura 5). La pequeña longitud de anclaje con la que fueron provistas las armaduras de flexión no permitía un adecuado desarrollo del efecto arco, fallando finalmente el anclaje de la armadura longitudinal en las vigas sin armadura de cortante, donde se veía progresar la fisura de cortante a lo largo de la armadura longitudinal en dirección a los apoyos.

Las curvas carga flecha pueden verse en la Figura 6, apreciándose el incremento de ductilidad que induce el empleo de fibras en la rotura a cortante. La viga PC-SR llega a romper por flexión. En vigas de SFRC, la carga máxima es un $20 \%$ mayor que la viga sin estribos de hormigón convencional, con dos veces su deformación bajo carga máxima. Se observa asimismo un incremento de ductilidad para todas las vigas de FRC, con respecto a las vigas de referencia, indicado por el notable incremento de deformabilidad de las vigas (p.e. mayor desplazamiento bajo cualquier nivel de carga) tanto bajo carga máxima como en el régimen de post-pico. A pesar de que la incorporación de fibras de polipropileno no incrementa el cortante último, la capacidad resistente a cortante de las vigas de FRC de polipropileno iguala a la de las de SFRC para grandes flechas (p.e. superior a $5 \mathrm{~mm}$ ).

Sin embargo, el cortante último de las vigas reforzadas con un $1 \%$ en volumen de fibras es siempre inferior al de las vigas con un mismo volumen de acero dispuesto en forma de estribos. Esto se debe principalmente a que la orientación de las fibras es aleatoria y sólo algunas de ellas se orientan favorablemente para oponer resistencia a la fisuración, mientras que todos los estribos son efectivos para resistir cortante.

\section{STRUCTURAL-SCALE TESTS RESULTS AND ANALYSIS}

The structural-scale beams were tested under shear loading through a four-point load configuration (Fig. 5), with a shear span to depth ratio equal to 2.4. The small anchorage length provided for the longitudinal reinforcement did not allow the development of the arch effect, which caused the failure by debonding of the flexural reinforcement in the case of beams without conventional shear reinforcement. The progress of the crack along the reinforcement and towards the supports could be visually observed.

Fig. 6 shows the load-deflection responses, where the shear ductility increase induced by fibre action can be observed. Beam PC?SR exhibited flexural failure. In SFRC beams, the maximum load is higher by approximately $20 \%$ of that of plain concrete, with twice the deflection at maximum load. An increase of ductility under shear loading can be clearly observed for all FRCs with respect to plain concrete beams, indicated by the significant increase in the deformability of the elements (i.e., a higher deflection at any load level) either at maximum load or along the post-peak regime. Though the incorporation of polypropylene fibres did not increase the ultimate load of the composite, the shear load-carrying capacity of the polypropylene FRC beams equalled that of SFRC for high deflection values, i.e. beyond $5 \mathrm{~mm}$.

Nevertheless, the load carrying capacity of the beams reinforced with $1 \%$ volume of fibres is always lower than that of the beams with the same volume of steel in the form of stirrups. This is attributed mainly to the distributed nature of the fibre reinforcement where only part of the fibres are favourably oriented to resist shear cracking, whereas all the stirrups actively prevent crack opening. 


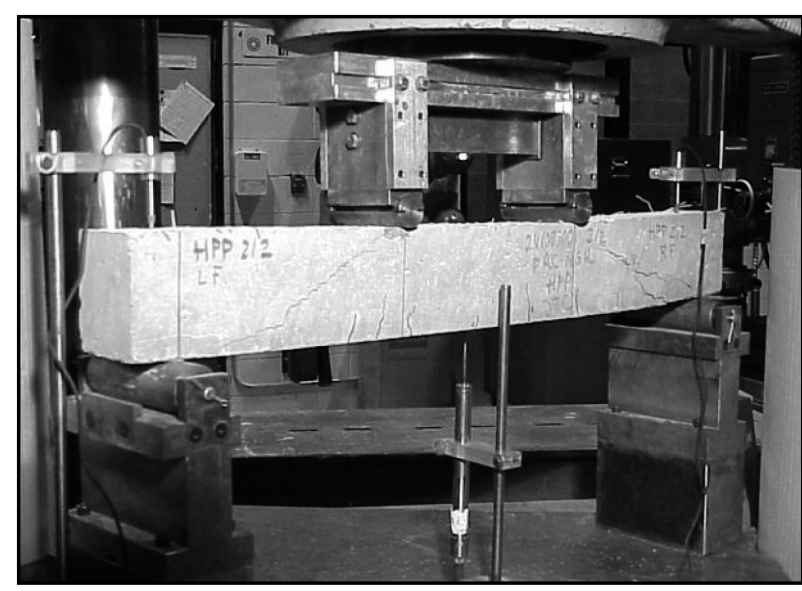

Figura 5. Ensayo de cortante.

Figure 5. Beam shear test.

En la Tabla 5, además de la carga última de cortante obtenida en los ensayos estructurales, se muestran los valores de cortante deducidos de la aplicación de los métodos de cálculo propuestos por Al-Taan y Al-Feel (2) (denominados A\&A); Campione y Mindess (4) (denominados C\&M); RILEM (5); y Dupont y Vandewalle (6) (denominados D\&V). Debido a que estos métodos utilizan cada uno diferentes parámetros obtenidos de diversos ensayos, algunos de éstos han sido extrapolados en función de resultados obtenidos en otros estudios. Así, la resistencia a flexotracción equivalente que emplea el método de RILEM se obtuvo a partir del índice I20 de la normativa ASTM siguiendo el estudio realizado por Dupont y Vandewalle (12). El método propuesto por Campione y Mindess (4) utiliza para el cálculo la resistencia a tracción por compresión diametral, la cual se estimó a partir de la resistencia media a compresión utilizando la fórmula propuesta por la EHE (13). El método de Dupont y Vandewalle (12) no separa las contribuciones del hormigón, de los estribos y de las fibras, sino que propone una formulación que las engloba.

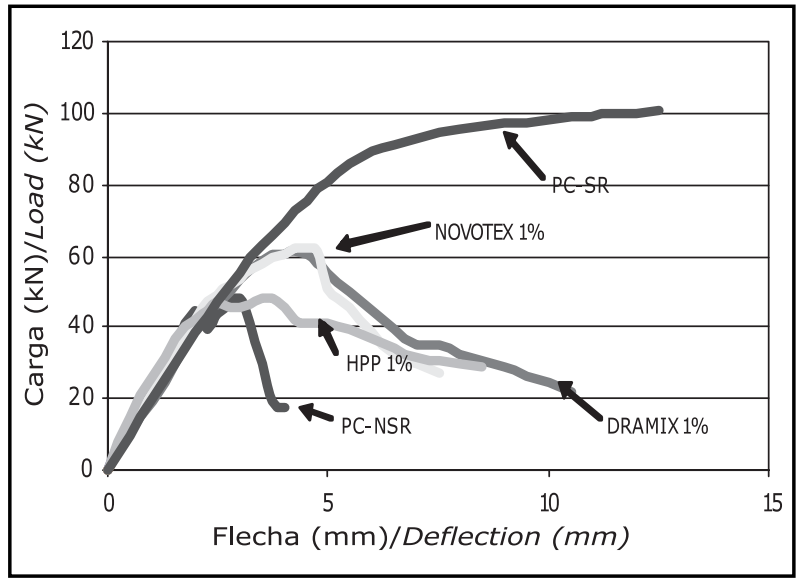

Figura 6. Diagrama carga flecha en el ensayo de cortante. Figure 6. Load-displacement response.

Table 5 presents the average ultimate load values obtained from tests, and those calculated from the formulae proposed by Al-Taan and Al-Feel (2) (denoted as A\&A); Campione and Mindess (4) (denoted as C\&M); RILEM (5); and Dupont and Vandewalle (6) (denoted as D\&V). Since each of these design methods use different toughness parameters obtained from different test configurations, some of the parameters had to be obtained from correlations with previous test results. Consequently, the equivalent flexural strength used by the RILEM method was obtained from the I20 index of the ASTM standard considering the study by Dupont and Vandewalle (12). The method proposed by Campione and Mindess (4) makes use of the tensile strength obtained from the splitting tension test, which was estimated from the compressive strength using the formula proposed by the Spanish Code (13). Note that the method by Dupont and Vandewalle (12) does not separate the contributions of the concrete, stirrups and fibres but proposes formulae for the material as a whole.

Tabla 5 / Table 5

Carga última de los ensayos estructurales y predicciones de la formulación. Test results and estimations from proposed formulae.

\begin{tabular}{|l|c|c|c|c|c|}
\hline Hormigón/Concrete & $\begin{array}{c}\text { Ensayo/ Test } \\
(\mathrm{kN})\end{array}$ & $\begin{array}{c}\text { RILEM } \\
(\mathrm{kN})\end{array}$ & $\begin{array}{c}\text { A \& A } \\
(\mathrm{kN})\end{array}$ & $\begin{array}{c}\text { C \& M } \\
(\mathrm{kN})\end{array}$ & $\begin{array}{c}\text { D \& V } \\
(\mathrm{kN})\end{array}$ \\
\hline PC-NSR & 50 & $*$ & $*$ & $*$ & $*$ \\
\hline DRAMIX 1\% & 62 & 48 & 62 & 54 & 39 \\
\hline NOVOTEX 1\% & 62 & 42 & 54 & 50 & 39 \\
\hline HPP 1\% & 50 & 32 & 54 & 52 & 37 \\
\hline PC-SR & 100 & $*$ & $*$ & $*$ & $*$ \\
\hline * Los métodos de diseño están sólo propuestos para FRC/The design methods are proposed only for FRC \\
\hline
\end{tabular}


Se puede observar en la Tabla 4 que el método propuesto por Al-Taan y Al-Feel (2)consigue estimar la resistencia a cortante de manera bastante precisa, seguido por el método de Campione y Mindess (4); en ambos casos obteniendo una muy buena estimación incluso en el caso de FRC con fibras de polipropileno. Los resultados de esta serie de ensayos son algo conservadores comparados con las predicciones de los métodos propuestos por RILEM y Dupont y Vandewalle.

\section{CONCLUSIONES}

Se observó que el hecho de añadir fibras a la mezcla incrementa la tenacidad del hormigón, tanto a flexión como a compresión, como queda reflejado en los índices de tenacidad propugnados por la normativa ASTM y la JSCE. Este incremento de tenacidad del material, se transforma en un incremento de ductilidad en el comportamiento a cortante, llegando en las vigas con refuerzo de fibras de acero a incrementos muy significativos de deformabilidad.

En vigas de SFRC, la carga máxima es un $20 \%$ mayor que la viga sin estribos de hormigón convencional, con dos veces su deformación bajo carga máxima. A pesar de que la incorporación de fibras de polipropileno no incrementa el cortante último, la capacidad de resistencia a cortante de las vigas de FRC de polipropileno iguala a la de las de SFRC para grandes flechas (p.e. superior a $5 \mathrm{~mm}$ ).

La respuesta de las vigas de FRC con un $1 \%$ en volumen de fibras aparece como inferior a la de las vigas con el mismo volumen de acero incorporado en forma de cercos.

Los métodos de cálculo aplicados resultan adecuados para predecir la resistencia última a cortante de vigas de sección rectangular, apareciendo como suficientemente precisos al permitir la utilización de parámetros extrapolados en sus fórmulas.

\section{AGRADECIMIENTOS}

Los autores agradecen al Ministerio de Educación y Ciencia español la financiación recibida a través de los proyectos BIA2005-09229-C02-01/02, BIA2006-15471C02-01/02, MAT2003-05530 and PSE 11-2005/PSS380000-2005-12/2007-1. Uno de los autores disfrutó de una beca de Formación del Profesorado Universitario del Ministerio de Educación español, que financió la estancia en la Universidad de British Columbia, donde se realizaron los ensayos.
Regarding the proposed design recommendations, Table 4 shows that the formulation proposed by Al-Taan and Al-Feel (2) leads to a satisfactory precise estimation of the shear capacity, followed by that of Campione and Mindess (4); in both cases a fairly good estimation is obtained even for the case of the polypropylene FRC. The test results show that the recommendations proposed by RILEM (5) and Dupont and Vandewalle (6) considerably underestimate the shear capacity of the beams.

\section{CONCLUSIONS}

It has been observed that the incorporation of fibres to the mix increases the material toughness both in tension or compression, as indicated by the toughness indexes of the JSCE and ASTM standards. The material toughness increase results in higher shear strength of the concrete and better deformability, i.e. the deflection at maximum load is significantly higher for FRC beams than plain concrete specimens.

In SFRC beams, the maximum load increased by approximately $20 \%$ with respect to the plain concrete beams, with twice the deflection at maximum load. Though the incorporation of polypropylene fibres did not increase the ultimate load of the composite, the shear load-carrying capacity of polypropylene FRC beams equalled that of the $S F R C$ for high deflection values, i.e. beyond $5 \mathrm{~mm}$.

The response of beams reinforced with $1 \%$ by volume of fibres is always lower than the beams containing the same volume of steel in the form of stirrups.

The applied design formulations are found to be adequate for estimating the ultimate shear capacity of rectangular cross-section beams, being sufficiently robust even when all the relevant material data may not be available.

\section{ACKNOWLEDGEMENTS}

The authors acknowledge Spanish Ministry of Education and Science for the financial support received under the research projects BIA2005-09229-C02-01/02, BIA200615471-C02-01/02, MAT2003-05530 and PSE 112005/PSS-380000-2005-12/2007-1. One of the authors benefited from a scholarship from the Spanish Ministry of Education (Scholarship for the Formation of University Professors) which funded the stay in the University of British Columbia, where the tests were carried out. 


\section{BIBLIOGRAFÍA / BIBLIOGRAPHY}

(1) Almansa, E. M. and Canovas, M. F.: "Mix design of steel fiber reinforced concrete" Mater Construcc, Vol. 47, No 247-48 (1997), pp. 11-26.

(2) Al-Taan, S.A. and Al-Feel, J.R. "Evaluation of Shear Strength of Fiber-Reinforced Concrete Beams", Cement and Concrete Composites, Vol. 12 (1990) pp. 87-94. http://dx.doi.org/10.1016/0958-9465(90)90045-Y

(3) Casanova, P. and Rossi, P., "Analysis and Design of Steel Fiber Reinforced Concrete Beams", ACI Structural Journal, Vol. 94 , No. 5 (1997) pp. 595-602.

(4) Campione, G. and Mindess, S. "Fibers as Shear Reinforcement for High Strength Reinforced Concrete Beams Containing Stirrups", Proceedings International Workshop on High Performance Cement Composites, HPFRCC3, Mainz, Germany (Eds. H.W. Reinhardt y A.E. Naaman) RILEM Publications S.A.R.L., Vol. 6 (1999) pp. 519-529.

(5) RILEM 'Test and design methods for steel fiber reinforced concrete: s-e Design Method', RILEM TC 162-TDF Recommendations, Materials and Structures, Vol. 33 (2000) pp. 75-81. http://dx.doi.org/10.1007/BF02484159

(6) Dupont, D. and Vandewalle, L., 'Shear Capacity of Concrete Beams Containing Longitudinal Reinforcement and Steel Fibers' Proceedings ACI Fall Convention 2000, ACI-SP, American Concrete Institute, Detroit, USA. (2000).

(7) CNR-DT 204/2006, Guide for design, execution, and control of fibre reinforced concrete structures (Istruzioni per la Progettazione, l'Esecuzione ed il Controllo di Stutture di Calcestruzzo Fibrorinforzato), National Council of Research (Consiglio Nazionale delle Ricerche), Italy. (in Italian) (2006).

(8) RILEM "Test and design methods for steel fiber reinforced concrete: Bending Test" RILEM TC 162-TDF Recommendations, Materials and Structures, V. 33 (2000) pp. 3-5. http://dx.doi.org/10.1007/BF02481689

(9) JSCE-SF5, "Method of Tests for Compressive Strength and Compressive Toughness of Steel Fibre Reinforced Concrete", Recommendation for Design and Construction of Steel Fibre Reinforced Concrete. JSCE (1984).

(10) ASTM C 1018 - 97, "Standard Test Method for Flexural Toughness and First-Crack Strength of Fiber-Reinforced Concrete" (1998)

(11) Altun, F.; Haktanir, T.A. "Comparative experimental study of steel fibre-additive reinforced concrete beams" Mater Construcc, Vol. 54, No 276 (2004), pp. 5-15.

(12) Vandewalle, L. and Dupont, D., "Comparison between the Bending Tests According to RILEM, NBN, JCI, ASTM and the Uniaxial Tension Test", Sub-task 2.3, Brite-Euram European Project BRPR.CT98.0813, "Test and design methods for steel fibre reinforced concrete". (2001).

(13) EHE, Spanish Building Code ("Instrucción de Hormigón Estructural"), Ministerio de Fomento, Madrid. (in Spanish) (1999). 
